Rosse, CG \& Melim, LMC. (2020). Fundamentals of cooperative learning, didactic strategies and the teaching of Natural Sciences. Research, Society and Development, 9(7):1-27, e157973611.

Fundamentos da aprendizagem cooperativa, estratégias didáticas e o ensino de Ciências Naturais

Fundamentals of cooperative learning, didactic strategies and the teaching of Natural Sciences

Fundamentos del aprendizaje cooperativo, estrategias didácticas y la enseñanza de las Ciencias Naturales

Recebido: 23/04/2020 | Revisado: 27/04/2020 | Aceito: 29/04/2020 | Publicado: 01/05/2020

Cássio Gomes Rosse

ORCID: https://orcid.org/0000-0002-0718-3116

Fundação Oswaldo Cruz (Fiocruz / RJ), Brasil

E-mail: cassiogrosse@gmail.com

Leandra Marques Chaves Melim

ORCID: https://orcid.org/0000-0002-8446-8879

Colégio Técnico da Universidade Federal Rural do Rio de Janeiro, Brasil

E-mail: lemelim@gmail.com

\title{
Resumo
}

A aprendizagem cooperativa é uma prática instrucional na qual os estudantes realizam atividades em pequenos grupos, compartilham recursos e ideias em condições de interdependência positiva. Trata-se de uma prática estudada e avaliada em muitos países, nos mais diversos contextos educacionais: desde a idade pré-escolar até a pós-graduação, podendo ser aplicada nas mais diversas disciplinas e currículos escolares. Contraditoriamente, a aprendizagem cooperativa ainda é uma prática incipiente no Brasil. Neste trabalho, de caráter estritamente teórico, foi realizada uma caracterização dos fundamentos da aprendizagem cooperativa, suas condições para implementação e as estratégias de ensino que se fundamentam nessa prática. Foram feitos levantamentos a respeito da eficácia dessa prática em relação a outras já tradicionalmente estabelecidas, como a competição e o individualismo. No artigo, destacam-se considerações a respeito do papel docente enquanto orientador dessa 
prática. Ao final, buscou-se associar aspectos da aprendizagem cooperativa em prol de uma área específica: o ensino de Ciências.

Palavras-chave: Aprendizagem cooperativa; Estratégias cooperativas; Ensino de Ciências.

\begin{abstract}
Cooperative learning is an instructional practice in which students perform activities in small groups, share resources and ideas in conditions of positive interdependence. It is a practice studied and evaluated in many countries, in the most diverse educational contexts: from preschool to post-graduation, and it can be applied in the most diverse disciplines and school curricula. Contradictorily, cooperative learning is still an incipient practice in Brazil. In this work, which is strictly theoretical, a characterization of the fundamentals of cooperative learning, its conditions for implementation and the teaching strategies that are based on this practice were carried out. Surveys were made about the effectiveness of this practice in relation to others already traditionally established, such as competition and individualism. In the article, considerations about the teaching role as a guide for this practice are highlighted. At the end, we sought to associate aspects of cooperative learning in favor of a specific area: science teaching.
\end{abstract}

Keywords: Cooperative learning; Cooperative strategies; Science Teaching.

\title{
Resumen
}

El aprendizaje cooperativo es una práctica educativa en la que los estudiantes realizan actividades en grupos pequeños, comparten recursos e ideas en condiciones de interdependencia positiva. Es una práctica estudiada y evaluada en muchos países, en los contextos educativos más diversos: desde la edad preescolar hasta la postgrado, y puede aplicarse en las más diversas disciplinas y currículos escolares. Contradictoriamente, el aprendizaje cooperativo sigue siendo una práctica incipiente en Brasil. En este trabajo, que es estrictamente teórico, se realizó una caracterización de los fundamentos del aprendizaje cooperativo, sus condiciones de implementación y las estrategias de enseñanza que se basan en esta práctica. Se realizaron encuestas sobre la efectividad de esta práctica en relación con otras ya establecidas tradicionalmente, como la competencia y el individualismo. En el artículo, se destacan las consideraciones sobre el papel de la enseñanza como guía para esta práctica. Al final, buscamos asociar aspectos del aprendizaje cooperativo en favor de un área específica: la enseñanza de las ciencias. 
Palabras clave: Aprendizaje cooperativo; Estrategias cooperativas; Enseñanza de las Ciencias.

\section{Aprendizagem Cooperativa}

A aprendizagem cooperativa é uma prática instrucional que começou a ganhar visibilidade no início dos anos de 1970. Em contraste com os métodos tradicionais, nos quais os estudantes realizam prioritariamente tarefas individualistas, na aprendizagem cooperativa os estudantes se ajudam mutuamente e são beneficiados por compartilharem ideias. Atualmente, esse tipo de aprendizagem é empregado em vários países, desde a pré-escola até as universidades (Gillies, 2014; Johnson \& Johnson, 2009).

A aprendizagem cooperativa tem sua origem nas teorias de interdependência social, introduzida por Kurt Koffka e Kurt Lewin na década de 1930 (Johnson \& Johnson, 2005). Os estudos de Koffka e Lewin inspiraram os trabalhos de Roger Johnson e David Johnson, que vêm estudando esta área e representam importantes referencias desde a década de 1960. (Johnson, Johnson, \& Holubec, 1994). Segundo os autores, a interdependência social existe quando a realização da meta de cada indivíduo depende do desempenho dos outros. A interdependência pode ser dividida em dois tipos: positiva e negativa.

A interdependência positiva (cooperação) existe quando há uma relação positiva e dependente entre os indivíduos: eles podem atingir um objetivo/meta apenas se os demais indivíduos com quem interagem também atinjam seus respectivos objetivos/metas. Essa interdependência promove interações positivas e os indivíduos ajudam e encorajam seus pares para completar suas tarefas em prol do objetivo do grupo. A interdependência negativa (competição) existe quando há uma relação negativa entre os pares: os indivíduos só podem atingir um objetivo/meta se os demais com os quais estão interagindo falhem na obtenção dos seus respectivos objetivos/metas. Esse tipo de interdependência também gera interações, porém elas acontecem por meio de resistências e desincentivos entre os pares. Além destas, a ausência de interdependência é caracterizada por atividades individualistas, na qual o desempenho de um indivíduo não afeta (positivamente ou negativamente) o desempenho dos demais (Johnson \& Johnson, 2009).

A maioria das atividades desenvolvidas em sala de aula é caracterizada por não apresentarem interações, sejam elas positivas ou negativas. Em pesquisa realizada com professores de Ciências e Biologia, os docentes indicaram que sempre ou quase sempre fazem uso de aulas expositivas. Além disso, os recursos didáticos mais utilizados foram o 
quadro/lousa e livros didáticos (Theodoro, Costa, \& Almeira, 2015). Nesse cenário, o aprendizado dos conteúdos, assim como sua avaliação tende a figurar-se de maneira essencialmente individual. Práticas que estimulam a interdependência negativa também são comuns. Muitas escolas, por exemplo, encorajam um ambiente competitivo por meio da criação de rankings de desempenho escolar (Afonso, 2009). Tais propostas podem desencorajar, desestimular e aumentar conflitos entre os estudantes, especialmente entre aqueles com baixo desempenho escolar.

Alguns relatos sugerem que estratégias individualistas podem desmotivar alguns alunos. De acordo com Felder, Richard \& Brent (2007) quando estudantes com dificuldade de aprendizado atuam individualmente, há maiores chances de que desistam de aprender, principalmente os conteúdos considerados mais difíceis. Quando os estudantes trabalham em grupo, eles persistem na tarefa, pois aqueles com mais facilidade em aprender ajudam e estimulam os que apresentam maiores dificuldades, estimulando-os a persistir nas tarefas. Ao ajudar seus pares, o indivíduo ainda pode perceber e preencher suas próprias lacunas no aprendizado (Ibidem, 2007).

As estratégias cooperativas de ensino são baseadas no pressuposto de que o aprendizado é mais eficaz quando os estudantes possuem a oportunidade de explicar suas ideias para os outros. No entanto, há diversas evidências apontando que atividades em grupo, por si só, não garantem um bom desempenho nas tarefas. É importante que seja uma atividade estruturada a partir da interdependência positiva entre os participantes, além da supervisão e acompanhamento dos professores (Herreid, 1998; Hwong, Caswell, Johnson, \& Johnson, 1993). A interdependência positiva gera maior responsabilidade individual, já que o desempenho de cada membro afeta diretamente o desempenho do grupo, criando forças de responsabilidade que estimulam o esforço e persistência na atividade. Isso pode contribuir para uma melhora no desempenho individual, mesmo se tratando de uma atividade realizada coletivamente (Johnson \& Johnson, 2009).

Em linhas gerais, a aprendizagem cooperativa existe quando os estudantes trabalham em grupo para dividir conhecimentos e atingir um objetivo comum, a partir de relações de interdependência positiva. Para se efetivar a aprendizagem cooperativa, são formados grupos pequenos, nos quais os participantes devem organizar seus recursos e tempo (Johnson, Johnson, \& Stanne, 2000). Vale ressaltar, que algumas estratégias de ensino cooperativo também incorporam a componente da competição em sua dinâmica, uma vez que pressupõem a cooperação dentro do grupo, mas envolvem competição entre diferentes grupos. 
Arason (2002) afirma que a aprendizagem cooperativa ainda incentiva os estudantes a se ouvirem, se engajarem e criarem laços afetivos, na medida em que cada membro possui uma importante tarefa a ser desenvolvida para o grupo. $\mathrm{Na}$ aprendizagem cooperativa, nenhum participante é capaz de atingir o objetivo do grupo de maneira isolada. Os objetivos são atingidos a partir da complementação das tarefas realizadas por cada um dos seus integrantes.

Diversos autores destacam que interdependência positiva, interações faca-a-face, responsabilidade individual, habilidades colaborativas e reflexões em grupo são elementos estruturantes para um ambiente de aprendizagem cooperativa. (Johnson \& Johnson, 2009; Ibidem, 2013; Tanner, Chatman, \& Allen, 2003; Zakaria \& Iksan, 2006). Esses elementos serão descritos a seguir na perspectiva desses autores.

\subsection{Interdependência Positiva}

Nas classes e grupos cooperativos, os estudantes trabalham em conjunto pelo sucesso de cada membro. Eles devem perceber que atitudes individuais ou competitivas não contribuem para o sucesso do grupo. Por isso, o ambiente de trocas, incentivo mútuo e diálogo são essenciais. No entanto, estruturar atividades cooperativas de ensino não é uma tarefa trivial. Para promover interdependência positiva, os professores precisam elaborar suas atividades de tal maneira que elas não possam ser realizadas individualmente ou apenas com esforço de parte do grupo (Laal, 2013; Tanner, Chatman, \& Allen, 2003).

Existem várias formas de gerar interdependência positiva entre membros de um grupo. Elas podem ser efetivadas pelos alunos apresentarem uma divisão clara de objetivos, recursos ou tarefas no interior do grupo. Essas formas de interdependência não são excludentes e, podem se sobrepor em uma atividade cooperativa. Algumas práticas são usuais entre aqueles que desenvolvem atividades cooperativas. Johnson \& Johnson (2009) destacam algumas:

- Divisão de atividades. Cada membro torna-se responsável pela resolução e compreensão de parte de um material;

- Divisão de papéis. Cada membro possui uma função única no interior do grupo. Enquanto uns intermedeiam discussões, outros produzem resumos e relatórios, por exemplo;

- Divisão de tarefas. Cada membro possui uma tarefa claramente definida a ser desempenhada no grupo. 
Outra prática usual entre aqueles que desenvolvem atividades cooperativas é unir as notas de cada membro em uma atividade desenvolvida coletivamente. Dessa maneira, a participação de cada um influencia diretamente na nota do grupo. Se um membro do grupo fica com nota baixa, todo grupo será afetado.

\subsection{Interações Face-a-face}

Os estudantes envolvidos em um ambiente de ensino cooperativo devem ter tempo e oportunidade para compartilhar ideias e opiniões oralmente, além de discutir os conceitos envolvidos na situação de aprendizagem. Tais contextos são importantes e devem ser estimulados pelo professor, que pode até inserir novas questões e controvérsias, de acordo com o andamento da aula. Para assegurar que tais momentos de discussões ocorram, pode ser solicitado aos grupos que produzam relatórios, ou que apresentem para o resto da classe as discussões realizadas. Essa divisão de tarefas é importante para facilitar as interações e assegurar a participação de todos os membros na dinâmica do grupo. Dessa forma, há um maior envolvimento a partir da responsabilidade individual dos alunos.

\subsection{Responsabilidade Individual}

Uma das vantagens da aprendizagem cooperativa é possibilitar aos estudantes o compartilhamento das ideias e habilidades de seus pares, de maneira a potencializar seu próprio aprendizado. Em situações cooperativas de ensino, cada estudante é responsável por seu progresso acadêmico, além do progresso do grupo como um todo. Dessa maneira, cada membro possui uma tarefa a ser desempenhada dentro do grupo e outras associadas a dinâmica do grupo. As responsabilidades individuais e do grupo podem ser estimuladas atribuindo notas e conceitos, tanto para os membros como para o grupo como um todo.

\subsection{Habilidades Colaborativas}

A aprendizagem cooperativa ainda possibilita o desenvolvimento de habilidades interpessoais, importantes não só no convívio escolar, mas também no ambiente de trabalho e na vida em sociedade de maneira geral. É possível destacar entre essas habilidades: ouvir e compreender diferentes pontos de vista, desenvolver espírito crítico frente a diferentes tipos de informação, defender e argumentar sobre opiniões baseadas em evidências, efetivar a 
(CC BY 4.0) | ISSN 2525-3409 | DOI: http://dx.doi.org/10.33448/rsd-v9i7.3611

comunicação, tomar decisões, analisar prioridades e resolver problemas (Johnson \& Johnson, 2009).

Uma pesquisa que avaliou o efeito de aulas cooperativas, observou que os estudantes do ensino primário se envolveram ativamente com atividades de leitura e discussão. Eles também se mostraram mais proativos na execução das tarefas e demonstraram maior vontade de realizá-las continuamente. A autora ainda percebeu que as crianças mais quietas e tímidas começaram a expressar suas opiniões e preferências no interior dos grupos cooperativos (LaCarrubba, 1993). Essas considerações são importantes e de grande relevância no contexto de ensino, dada heterogeneidade da maioria das salas de aula. $\mathrm{O}$ trabalho com grupos cooperativos pode favorecer que habilidades pouco estimuladas no contexto de educação formal possam ser desenvolvidas na escola.

\subsection{Processamento de Grupo}

Os grupos que trabalham cooperativamente por longos períodos devem avaliar constantemente seu progresso e precisam discutir ativamente como está o andamento das metas e objetivos do grupo e de seus membros. É igualmente importante que os grupos avaliem quais tipos de ações contribuem (ou não) para seu melhor desempenho. Esse processo de análise e processamento de grupo deve ser realizado durante as discussões em sala e deve ter acompanhamento direto do professor. Tanto os estudantes devem ter oportunidades de alertar ao professor sobre problemas intrínsecos ao grupo, assim como o professor deve promover feedbacks e sugerir soluções para resolução de possíveis conflitos.

O que é fundamental nesse item é que os estudantes devem ter tempo disponível para avaliar o andamento do próprio trabalho cooperativo. O processamento de grupo habilita aos estudantes trabalharem cooperativamente e a resolverem melhor seus conflitos, habilidade fundamental em qualquer ambiente, seja ele escolar, familiar ou de trabalho.

Para que uma atividade seja, portanto, classificada como cooperativa, ela deve atender aos cinco elementos previamente descritos (itens 1.1 ao 1.5 ). No entanto, nem toda atividade consegue atender a todos esses elementos em um único momento ou formato, especialmente as intervenções de curta duração. A literatura na área propõe algumas distinções entre a aprendizagem cooperativa e colaborativa, que serão descritas na seção a seguir. 


\section{Distinções entre Aprendizagem Cooperativa e Colaborativa}

Estudos variados sobre trabalhos em grupo adotam frequentemente os termos colaboração e cooperação, sem defini-los claramente ou estabelecer distinções entre eles. Os termos estão presentes em diversas áreas do conhecimento e podem ser tratados como sinônimos (Johnson et al., 2000), termos complementares (Torres \& Irala, 2007) ou distintos (Dillenbourg, 1999; Panitz, 1999). Apesar de os conceitos remeterem a metodologias ativas para se trabalhar em grupos, eles apresentam diferenças marcantes na natureza das atividades propostas e na maneira de conduzi-las.

A aprendizagem cooperativa pode envolver, entre outras características, a atribuição de funções e tarefas complementares para os indivíduos dentro de cada grupo, enquanto a aprendizagem colaborativa é caracterizada por processos relativamente não estruturados por meio dos quais os participantes negociam metas, definem os problemas, desenvolvem procedimentos e produzem conhecimento socialmente construído em pequenos grupos (Dillenbourg, 1999; Panitz, 1999). Segundo esses autores, a divisão de tarefas é elemento importante na aprendizagem cooperativa. A partir dela, cada membro torna-se responsável por realizar parte de uma atividade para seu grupo, que será efetivamente completa a partir da união das tarefas realizadas por cada membro.

Na aprendizagem colaborativa, os membros de um grupo fazem todo o trabalho em conjunto. Dillenbourg (1999) ainda sugere que na colaboração pode ocorrer alguma divisão espontânea de tarefas, mas que difere em aspectos importantes da cooperação, uma vez que é instável, não fixa e não é estabelecida uma divisão clara de tarefas, como na cooperação. Panitz (1999) também defende que a aprendizagem cooperativa é mais estruturada do que a aprendizagem colaborativa e envolve uma participação mais ativa dos professores. É comum admitir que na aprendizagem cooperativa os professores assumam um papel mais diretivo ao organizar o trabalho em grupo e que exista uma responsabilidade maior dos indivíduos dentro do grupo.

Este trabalho também distingue aprendizagem cooperativa e colaborativa, na perspectiva de que as estratégias cooperativas devem envolver um plano bem estruturado de trabalho em pequenos grupos, incluindo a divisão de tarefas para gerar interdependência positiva entre os membros. Nesse contexto, o papel do professor é fundamental no processo de construção de estratégias cooperativas e de condução de tais atividades. 


\section{O papel do Professor na Aprendizagem Cooperativa}

Apesar de o paradigma central da aprendizagem cooperativa estar centrado na mudança de postura dos estudantes, o papel do professor não deve ser subestimado e sua postura deve estar de acordo com as próprias características dessa modalidade de ensino. Os estudantes não se engajam em propostas de diálogos construtivos espontaneamente, não costumam fazer perguntas se não forem estimulados e não necessariamente utilizam seus conhecimentos prévios sem que haja alguma forma de orientação (King, 2002). É necessário que eles sejam instruídos e apoiados constantemente para que se envolvam com a nova proposta de atividade. Por isso, a postura do professor durante a aprendizagem cooperativa é decisiva.

Além de estruturar os grupos e tarefas a serem desempenhadas, o professor deve deixar claro o que ele espera que os estudantes realizem e como espera que eles se portem. Espera-se a adoção de um papel de facilitador. Dessa maneira, não é aconselhável ao professor fornecer informações prontas e/ou responder diretamente todas as perguntas dos grupos, pois dessa forma poderá inibir o trabalho cooperativo. Como facilitador, o professor procura estimular o diálogo e a troca de informações entre os membros, propor indagações, aprofundamentos e desafios, incentivar e valorizar o esforço dos estudantes, identificar e ajudar na resolução de conflitos. Com isso, ele estará ensinando seus estudantes a interagir, justificar e resolver problemas em um contexto de aprendizagem cooperativa (Gillies, 2016).

Como a maior parte das atividades que envolve a aprendizagem cooperativa é estruturada previamente, o professor tem mais oportunidades para avaliar, de uma maneira mais eficaz, a aprendizagem dos seus estudantes. No decorrer das atividades, ele pode andar pela sala de aula analisando atentamente a fala e reação dos estudantes, a maneira como os grupos cooperativos trabalham, suas possíveis dificuldades, avaliando qualitativa e subjetivamente o trabalho. Essa postura é difícil de ser adotada em um contexto de ensino tradicional, no qual ocorre a centralidade do professor durante a instrução. Na literatura referente à aprendizagem cooperativa, o professor tem disponível uma série de estratégias que apresentam abordagens diferenciadas e podem ser aplicadas em diferentes contextos de aprendizagem, descritas a seguir. 


\section{Estratégias de ensino cooperativo}

Existe na literatura uma ampla gama de estratégias cooperativas de ensino. Apesar de todas assumirem pressupostos apresentados anteriormente, elas diferem na maneira de estruturar as atividades, na formação e organização dos grupos e na utilização de diferentes dinâmicas. A seguir, será apresentado, de forma resumida, as estratégias de aprendizagem cooperativa mais utilizadas na área de ensino da maneira como foram concebidas pelos seus autores. Os docentes podem utilizar essas estratégias em suas práticas, mas também podem adaptá-las a sua realidade escolar, assim como ao seu público-alvo. Entre as estratégias de ensino cooperativo mais conhecidas, destacam-se: Student Teams-Achievement Division (STAD), Teams-Games-Tournament (TGT), Jigsaw, Think-Pair-Share e Group Investigation. Elas serão descritas a seguir, segundo os referenciais de Slavin (2010), Li \& Lam (2013), Slavin (2010).

\subsection{Student Teams-Achievement Division (STAD)}

O método STAD foi proposto pelo psicólogo Rober Slavin em 1977. Essa estratégia cooperativa é utilizada em diversas áreas do conhecimento, como Matemática, Artes, Estudo Sociais e Linguagens. Sua metodologia pode ser dividida em quatro fases/etapas importantes e complementares.

1. Apresentação - o professor apresenta os materiais e os recursos que serão utilizados durante a aula para toda turma. Ele também apresenta os objetivos das atividades propostas.

2. Discussão em grupo - são formados grupos que podem ser organizados de acordo com o desempenho, habilidades, gênero, etnia, entre outros critérios. Os grupos estudam o material apresentado e realizam as tarefas propostas. Os estudantes trabalham cooperativamente e asseguram que todos os membros realizaram suas atividades e foram capazes de aprender o conteúdo. Eles podem questionar uns aos outros, fazer perguntas e compartilhar informações durante esse momento, uma vez que estão em uma condição de interdependência.

3. Teste - todos os estudantes realizam um teste individual sobre o material estudado. Neste momento, a dinâmica adotada deixa de ser cooperativa e passa a ser individual: os estudantes dos grupos não podem mais se comunicar. Usualmente são utilizadas questões de múltipla-escolha. O desempenho de cada estudante é comparado com seu 
Research, Society and Development, v. 9, n. 7, e157973611, 2020

(CC BY 4.0) | ISSN 2525-3409 | DOI: http://dx.doi.org/10.33448/rsd-v9i7.3611

próprio desempenho em testes anteriores à atividade. De acordo com a melhora da sua nota, o estudante recebe uma pontuação.

4. Reconhecimento - o professor pode somar a pontuação dos membros e avaliar, comparativamente, o desempenho dos grupos. É interessante notar, que o grupo com melhor desempenho não necessariamente é aquele que possui maiores notas, mas aqueles que conseguiram melhor progressão em suas notas, que será refletido em sua pontuação. O reconhecimento pode ser atribuído ao grupo com maior pontuação, ou para aqueles que conseguiram atingir uma meta de pontuação previamente definida.

$\mathrm{O}$ ciclo com as quatro etapas costuma durar de três a cinco aulas. $\mathrm{O}$ sucesso desse método cooperativo está associado à importância do envolvimento e real participação de cada membro na atividade proposta pelo professor. Dessa maneira, eles irão se esforçar ao máximo para melhorar seu desempenho individual, assim como ajudarão seus pares na sua melhora. $\mathrm{O}$ STAD oferece oportunidades iguais de vitória, mesmo para grupos formados por alunos que tenham mais dificuldade.

Outro aspecto interessante desse método é que ele pode ser considerado uma estratégia mista. Apesar de existir interdependência positiva dentro do grupo, há interdependência negativa, efetivada pela competição entre grupos para obter maior pontuação.

\subsection{Teams-Games-Tournament (TGT)}

Esta estratégia de ensino cooperativo, também desenvolvida pelo psicólogo Robert Slavin em 1978, apresenta as mesmas características da anterior, porém os testes são substituídos por torneios que não utilizam o mesmo critério em relação ao desempenho. $\mathrm{O}$ TGT também pode ser dividido em quatro etapas. As duas primeiras e a última são as mesmas do método STAD e, por isso, não serão descritas novamente.

No terceiro momento, denominado torneio (que substitui o teste da estratégia STAD) são formados novos grupos de três a quatro estudantes. Cada membro de um novo grupo é oriundo dos grupos anteriores. Os grupos são formados de acordo com a similaridade de notas obtidas em torneios anteriores, formando um grupo homogêneo que pode competir em igualdade de condições. Uma maneira de estruturar os torneios é disponibilizar um conjunto de cartas contendo perguntas sobre o assunto estudado. $\mathrm{O}$ jogador sorteia uma das cartas e responde à pergunta feita. Caso acerte, ele ficará com a carta. Os demais membros do grupo podem desafiar a resposta de seu par. Se respondê-la corretamente, o desafiante fica com a 
carta. Caso erre, o desafiante deve perder uma de suas cartas conquistadas durante a dinâmica. O jogo termina quando todas as cartas com perguntas acabam. Ao final, os jogadores serão pontuados de acordo com o número de cartas coletadas. A pontuação recebida por cada jogador será contabilizada para seu grupo de origem. O grupo que obtiver maior pontuação é considerado vencedor.

Diferentemente do método STAD, que valoriza a melhora no desempenho nas atividades, na dinâmica envolvendo o TGT é valorizado a performance de cada jogador, ao competir com seus pares em um grupo homogêneo. A cooperação é estimulada a medida em que o bom desempenho de cada jogador irá contribuir decisivamente na nota obtida pelo grupo de origem. Assim como a estratégia anterior, o TGT envolve interdependência positiva nos grupos iniciais, mas promove interdependência negativa entre os jogadores nos grupos formados posteriormente.

\subsection{Jigsaw}

O método do Jigsaw foi um dos primeiros métodos de ensino totalmente cooperativo. Essa estratégia foi inicialmente proposta Elliot Aronson na década de 1970 e foi aprimorada ao longo do tempo. Atualmente é uma das estratégias cooperativas mais conhecidas e é utilizada nas mais diversas áreas e disciplinas. A estratégia envolve a divisão dos materiais de ensino em partes. Assim como nas demais estratégias, são formados grupos de quatro a cinco estudantes. O Jigsaw pode ser dividido em momentos diferentes e complementares:

1. Leitura - o professor divide os alunos em pequenos grupos (grupo de origem) e cada membro do grupo recebe um texto com parte do assunto que será tratado. Após a formação dos grupos de origem, cada estudante deve ler seu material sozinho, seja em sala de aula ou em casa. Cada aluno receberá uma parte da atividade proposta para o grupo, garantindo assim a divisão de tarefas. Dessa maneira, cada membro terá um importante e insubstituível papel a ser desempenhado.

2. Grupo de experts - posteriormente são formados novos grupos (grupos de experts), constituídos por membros de grupos de origem que receberam a mesma parte do texto. Os grupos de experts são formados para que os estudantes possam discutir detalhadamente o material seus respectivos materiais de aprendizagem. Para facilitar a discussão, algumas questões norteadoras podem ser apresentadas pelo professor. Os experts ainda são estimulados a realizarem anotações sobre o que foi discutido para que possam ensinar aos demais membros do seu grupo de origem. 
3. Relatório dos grupos de origem - após terem discutido detalhadamente cada parte da atividade nos grupos de experts, os estudantes voltam aos seus grupos de origem para realizar todas as atividades coletivas propostas. Eles são estimulados a explicar uns aos outros os fragmentos das atividades estudados nos grupos de experts. O professor pode promover uma breve discussão com toda classe com o intuito de esclarecer possíveis dúvidas ou aprofundar algumas questões. O objetivo é entender primeiro as partes, para posteriormente se ter uma compreensão do todo.

Diferentemente das estratégias anteriores, a estratégia de ensino Jigsaw pode ser considerada estritamente cooperativa, pois envolve interdependência positiva gerada a partir da divisão de tarefas e não envolve interdependência negativa no interior do grupo ou entre grupos.

\subsection{Think-Pair-Share}

Essa estratégia de ensino cooperativo pode ser utilizada em diferentes áreas do conhecimento, pois ela apresenta uma estrutura simples e facilmente adaptável pelo professor aos mais diversos conteúdos de ensino. Uma maneira simples de utilizar a metodologia consiste em, pelo menos, três momentos:

1. Inicialmente os estudantes realizam uma atividade individual. Geralmente essa atividade envolve a leitura e compreensão de um material e os estudantes são desafiados a responderem perguntas propostas pelo professor sem qualquer forma de consulta, seja dos demais alunos ou do professor.

2. Em seguida os estudantes formam pares e podem compartilhar suas resoluções, dificuldades ou conclusões até que os alunos cheguem a um consenso. Durante a troca, eles podem trabalhar importantes habilidades colaborativas, o que os deixará mais envolvidos com a atividade.

3. Os estudantes compartilham suas respostas com toda a turma. Dessa forma, cada par será ouvido e uma discussão pode ser realizada pelo professor ao final de todas as falas.

\subsection{Group Investigation}

Nessa estratégia de ensino cooperativo os estudantes trabalham em grupo e têm a responsabilidade de criar, desenvolver e apresentar um projeto de pesquisa. Group 
Investigation é uma estratégia que oferece bastante liberdade aos grupos, que podem decidir o tópico a ser investigado e a maneira como irão conduzi-lo. Nessa estratégia é interessante que sejam apresentados problemas de investigação abertos, que podem apresentar diversas soluções. Trabalhar com projetos abertos possibilita aos estudantes desenvolver uma série de habilidades, como a proposição de hipóteses, realização de buscas adequadas, desenvolvimento do pensamento abstrato, preparar apresentações, além de várias outras habilidades cooperativas desenvolvidas no decorrer do trabalho em grupo. Considerando sua proposta mais ampla e menos estruturada (mais próximo, inclusive, da definição de aprendizagem colaborativa), o Group Investigation pode ser utilizado nas mais diversas áreas, porém alguns passos devem ser obedecidos.

1. Formação dos grupos e escolha de tópicos - o professor apresenta para toda classe uma temática ampla ou uma unidade. Durante a apresentação, alguns tópicos são sugeridos dentro de uma mesma unidade ou temática. Os estudantes que estejam interessados em trabalhar em um mesmo tópico podem formar um mesmo grupo. Os grupos formados possuem de quatro a seis estudantes.

2. Planejamento da investigação - os estudantes devem planejar o que eles desejam pesquisar no contexto do tópico escolhido. É interessante que eles proponham uma pergunta de pesquisa que norteará o trabalho. Em seguida, o grupo deve planejar os meios para que possam realizar a investigação. Eles devem propor uma metodologia para o projeto (onde realizar suas buscas, selecionar materiais ou equipamentos necessários, elaborar questionários, realizar entrevistas, entre outros).

3. Realização da investigação - após a definição do tópico e da pergunta que norteará a investigação, os estudantes devem colocar em prática o que foi proposto pelo grupo. Apesar de cada membro ter sua atribuição, o grupo deve reunir-se periodicamente para trabalhar de maneira coletiva. Dessa forma, eles realizam um trabalho cooperativo e os pares se ajudam mutuamente. Em alguns momentos o professor pode interferir para resolver possíveis conflitos e estimular o trabalho cooperativo. Eles ainda devem ser estimulados a escrever relatórios do trabalho realizado por cada membro.

4. Planejamento da apresentação - realizada a investigação, os grupos devem apresentar seus resultados aos demais. Eles devem selecionar cuidadosamente os dados a serem apresentados da forma mais didática possível, de maneira que os outros grupos possam entender todo projeto e investigação realizada por seus pares. O professor e 
um comitê de estudantes formados por membros de todos os grupos podem ajudar dando dicas para elaboração das apresentações.

5. Apresentação - os grupos realizam suas apresentações para toda classe, que deve se manter atenta a todos os projetos. Perguntas elaboradas por cada grupo podem ser incorporadas na forma de um teste a ser respondido por todos.

6. Avaliação - o professor deve avaliar o desempenho dos grupos e de cada membro. Um teste pode ser realizado contendo questões de cada um dos projetos apresentados. As questões podem ser elaboradas pelos próprios grupos. O professor ainda poderá realizar avaliações ao longo de todas as etapas anteriores a partir de sua observação no desenvolvimento dos projetos. Ele ainda pode propor um relatório final como forma de avaliação ou pedir para os estudantes realizarem auto avaliações.

Apesar de as estratégias cooperativas descritas serem interessantes, se faz necessário avaliar sua eficácia em termos didáticos pedagógicos, especialmente comparadas com as estratégias comumente utilizadas nas salas de aula. Na próxima seção serão destacados resultados de pesquisa que avaliam o efeito de estratégias cooperativas de ensino.

\section{Avaliação de estratégias cooperativas}

No início dos anos 1980, os irmãos Johnson realizaram uma meta-análise que avaliou o efeito de estratégias cooperativas, competitivas e individuais. Ela foi constituída de 122 estudos independentes realizados em escolas norte americanas. Os resultados indicaram que as estratégias cooperativas foram mais eficazes do que as estratégias competitivas e individuais. Estratégias cooperativas que envolviam competição entre grupos também apresentaram resultados significativamente melhores do que a estratégias individuais. Não foram identificadas diferenças entre estratégias estritamente cooperativas e estratégias cooperativas que envolviam competição entre grupos. No entanto, a quantidade de estudos para tal comparação, segundo os autores, era insuficiente (Johnson et al., 1981).

Outras revisões sistemáticas publicadas posteriormente confirmaram os resultados positivos das estratégias cooperativas. Slavin (1989) avaliou 60 estudos que compararam estratégias cooperativas com grupos controles, que geralmente eram formados por estudantes que realizavam atividades individuais. Os critérios de inclusão indicados pelo autor foram: os dois grupos comparados deveriam estudar o mesmo material, os estudantes deveriam ser aleatoriamente associados à intervenção ou grupo controle, não poderia existir diferenças iniciais entre os dois grupos, as intervenções deveriam durar, pelo menos, quatro semanas e 
deveriam existir, no mínimo, dois grupos experimentais e dois grupos controles, com o objetivo de minimizar o efeito de outras variáveis. Os resultados da análise de desempenho foram favoráveis para as estratégias cooperativas em $72 \%$ dos estudos. Não houve diferenças entre os dois grupos em $13 \%$ deles, enquanto que em $15 \%$ dos trabalhos os grupos controle obtiveram melhores resultados.

No início dos anos 2000, uma segunda meta-análise proposta pelos irmãos Johnson avaliou 158 trabalhos em relação à eficácia dos métodos cooperativos. A variável independente analisada no estudo foram os diferentes métodos cooperativos de ensino. Os autores identificaram oito diferentes métodos, alguns deles apresentados nesse trabalho. Eles foram comparados a métodos competitivos ou individuais. Todos os métodos cooperativos relatados nos trabalhos tiveram impacto positivo no desempenho dos estudantes. Além disso, as estratégias cooperativas obtiveram melhores resultados quando comparadas com estratégias individuais ou competitivas. É interessante destacar que mesmo as estratégias cooperativas que apresentam competição entre grupos (STAD e TGT) apresentaram desempenho significativamente superior as estratégias puramente competitivas ou individuais. Esses resultados indicam que a competição pode ser estruturada dentro de um contexto de aprendizagem cooperativa sem, entretanto, afetar o desempenho dos estudantes (Johnson et al., 2000).

Demais pesquisas de cunho primário publicados posteriormente validaram a eficácia das estratégias cooperativas frente às estratégias individualistas. Essas pesquisas foram realizadas em países que apresentam realidades educacionais bastante distintas. Além disso, os estudos sobre a eficácia dessas estratégias apresentam resultados favoráveis desde os primeiros anos de escolaridade até a pós-graduação, nas mais variadas disciplinas e currículos (Acar \& Tarhan, 2008; Anderson, Mitchell, \& Osgood, 2005; Ebrahim, 2012; Eymur \& Geban, 2016; Fernández-Santander, 2008; Genc, 2016; Gupta, Jain, \& Pasrija, 2014; Rothenberg, McDermott, \& Martin, 1998; Thomas \& Kothari, 2015).

Apesar de já existir um consenso na literatura sobre os efeitos positivos da aprendizagem cooperativa no desempenho dos estudantes, ainda há controvérsias sobre o porquê e como os métodos de aprendizagem cooperativos produzem tais efeitos (Slavin, 2015). Um dos principais motivos atribuídos ao sucesso das estratégias cooperativas é a motivação proporcionada, sendo esse o elemento central de qualquer processo de aprendizagem. O engajamento nas atividades, a persistência, a articulação com demais membros do grupo são gerados, a priori, a partir da motivação com a proposta de ensino (Slavin, 2015). 
Nas estratégias cooperativas que envolvem competição entre grupos, como é o caso das estratégias STAD e TGT, uma parte substancial da motivação associada à atividade provêm da componente competitiva. Assumindo algumas condições, a competição pode ser construtiva. Para tanto, ela precisa ser estruturada dentro de um contexto colaborativo, no qual são especificadas suas condições (de que maneira ela deve ocorrer e quais são seus limites), os critérios para vitória, as regras estipuladas e os árbitros. Os participantes devem competir em grupos homogêneos, no diz respeito às suas habilidades para que os competidores consigam perceber que todos possuem iguais chances de vitória. Eles devem ter condições para avaliar o progresso da competição (quem está à frente ou abaixo e quanto esforço é necessário para vitória). A competição deve ser encarada com certa trivialidade e os envolvidos devem ter humildade nos momentos de vitória e derrota. Além disso, os competidores não devem generalizar os resultados da competição para outros aspectos da vida social (Johnson \& Johnson, 2013).

Em síntese, há vasta evidência científica sobre a eficácia de estratégias cooperativas de ensino. Os estudos reportados nessa seção foram realizados em diferentes países, em diferentes contextos educacionais. A seguir será destacado sobre a relação da aprendizagem cooperativa com uma área específica: a de Ciências Naturais.

\section{Aprendizagem Cooperativa e o Ensino de Ciências Naturais}

A aprendizagem cooperativa pode ser um importante recurso no ensino de Ciências Naturais. Considerando sua natureza investigativa, as disciplinas científicas oferecem meios a partir dos quais podem ser estruturadas atividades cooperativas. Um dos principais benefícios dessa estratégia de ensino é a promoção da discussão, um elemento central para o aprendizado de Ciências Naturais. Relatos de professores que implementaram atividades cooperativas nessa área destacam que a prática promoveu a discussão em grupos e desenvolveu importantes habilidades, como o compartilhamento e troca de ideias, além de possibilitar que os conteúdos científicos fossem trabalhados com maior aprofundamento (Gillies \& Nichols, 2015).

Colosi \& Zales (1998), por exemplo, enfatizam o potencial do método cooperativo Jigsaw na melhoria das aulas de laboratório, uma vez que em diferentes grupos, os alunos podem lidar com diversas tarefas e usar diferentes técnicas laboratoriais para promover um ensino mútuo. Lord (2001) resume vantagens da aprendizagem cooperativa e indica que ela pode ajudar os alunos a perceberem as conexões entre a teoria e a vida real, com o benefício 
adicional do desenvolvimento de habilidades sociais, além de promover uma atitude positiva em relação à aprendizagem. Além disso, as estratégias cooperativas permitem que o processo de ensino e aprendizado seja focado na figura do aluno, aumentando a interação entre eles e mudando as relações estabelecidas em sala, tradicionalmente centradas na figura do professor (Day \& Bryce, 2013).

Apesar de diversos estudos relatarem os benefícios da aprendizagem cooperativa no desempenho acadêmico e nas atitudes dos estudantes, essa temática ainda é pouco explorada na área de ensino de Ciências Naturais. Silva, Teodoro \& Queiroz (2019) realizaram um levantamento de trabalhos publicados em revistas científicas nacionais e internacionais da área de ensino de Ciências, bem com trabalhos apresentados no Encontro Nacional de Pesquisa em Educação em Ciências (ENPEC). Segundo os autores, há um crescimento nas publicações ao longo dos anos, mas elas ainda são muito incipientes, principalmente no âmbito da pesquisa brasileira. A maioria dos trabalhos é voltada para o ensino de Química, sendo que a maioria deles destinado ao atendimento de estudantes do Ensino Médio. Internacionalmente, a aprendizagem cooperativa é mais utilizada no Ensino Superior. Os autores ponderam que a aprendizagem cooperativa, portanto, tem um alto potencial exploratório em todos os níveis de ensino.

Dois estudos de longa duração, conduzidos com alunos do Ensino Médio em diferentes países compararam o desempenho de alunos que foram submetidos a aulas regulares, com grupos que participaram de atividades cooperativas nas disciplinas de Física e Biologia. No estudo de Ho \& Boo (2007), o grupo experimental participou de atividades cooperativas, cujas temáticas eram eletricidade e circuitos elétricos, durante oito semanas. $\mathrm{O}$ grupo controle estudou o mesmo conteúdo com um professor que não utilizava tais atividades. Os resultados do pré e pós-teste indicaram que o grupo experimental obteve melhores resultados de desempenho. Além disso, os estudantes declararam que entenderam melhor os conteúdos de Física quando estudaram e discutiram as atividades em grupo (Ho \& Boo, 2007). Resultados semelhantes foram obtidos por Muraya \& Kimamo (2011). Os autores realizaram um estudo com 183 estudantes do ensino secundário do Quênia. Os autores buscaram determinar o efeito da aprendizagem cooperativa nas notas obtidas em exames de Biologia. $\mathrm{O}$ grupo experimental teve aulas ministradas por uma metodologia de aprendizagem cooperativa por cinco semanas e o grupo controle se manteve com as aulas regulares da classe. Os achados da pesquisa indicaram que houve diferenças estatisticamente significativas a favor do grupo experimental quando ambos foram submetidos aos exames da disciplina.

A aprendizagem cooperativa também apresenta resultados positivos no Ensino 
Superior. Armstrong, Chang, \& Brickman (2007) compararam o desempenho de estudantes, do primeiro período na disciplina de Introdução à Biologia, submetidos à aprendizagem cooperativa e ao formato tradicional por meio de exames. As classes eram constituídas por uma grande quantidade de alunos (acima de 250 por classe). Os resultados obtidos indicaram que os estudantes que aprenderam cooperativamente mostraram melhor progressão nos seus conhecimentos, ao longo da disciplina, quando comparados aos estudantes que tiveram o curso ministrado por meio de palestras. Os autores ainda discutem que a aprendizagem cooperativa pode ser efetiva mesmo em classes com grande contingente, pelo menos no contexto do Ensino Superior.

Para além da melhora no desempenho, outros autores relatam que o aprendizado de Ciências Naturais deve ser estruturado de maneira similar ao próprio processo de produção do conhecimento científico. Assim como cientistas trabalham de maneira cooperativa, cada qual contribuindo com conhecimentos e expertise da sua área de formação, os estudantes podem aprender os conteúdos científicos de maneira similar, compartilhando experiências e ideias (Tanner et al., 2003). Patchen \& Smithenry (2015) observaram mudanças na percepção de estudantes do ensino secundário sobre o que é Ciência, a partir da utilização de um currículo que utiliza dinâmicas colaborativas. Com a mudança, os estudantes perceberam a atividade científica como uma comunidade prática e colaborativa, distanciando a associação com uma atividade solitária, praticada por indivíduos com habilidades inatas.

A aprendizagem cooperativa também pode ser um método eficiente para o desenvolvimento da capacidade de resolução de problemas. Estudantes do Ensino Médio apresentados a essa modalidade de ensino demonstraram melhor capacidade de resolução de problemas envolvendo a disciplina de Genética, quando comparados com aqueles que o fizeram individualmente (Kirschner, Paas, Kirschner, \& Janssen, 2011). A aprendizagem cooperativa no ensino de Ciências Naturais pode contribuir também para formar estudantes capazes de trabalhar em grupo, não só no meio científico, mas em vários contextos da vida social. Após realizarem um curso de Nutrição, estudantes universitários relataram que a utilização da abordagem cooperativa pode ajudá-los em sua vida profissional. Eles destacaram que o trabalho em grupo e a resolução de problemas complexos são exigências constantes de suas carreiras (Goodell, Cooke, \& Ash, 2012).

Os trabalhos envolvendo a utilização de estratégias de ensino cooperativo no Brasil corroboram os resultados internacionais (Silva, Teodoro \& Queiroz, 2019). Fatareli, Ferreira, Ferreira \& Queiroz (2010) utilizaram o método cooperativo Jigsaw no ensino de reações químicas, para alunos do $2^{\circ}$ ano do Ensino Médio. A metodologia aplicada mostrou-se 
motivadora, uma vez que a maior parte da turma demonstrou interesse na execução da atividade, aprimorou a capacidade de comunicação escrita e contribuiu para a compreensão de conceitos. O trabalho de Oliveira, Kiouranis, Eichler \& Queiroz (2017) também utilizou a estratégia cooperativa Jigsaw e foi destinado a alunos bolsistas do Programa Institucional de Bolsas de Iniciação à Docência (PIBID) do Curso de Química. A atividade foi realizada em três encontros de duas horas de duração cada e abordou aspectos químicos, econômicos e sociais sobre o chocolate. Por meio da utilização de pré e pós-testes, os autores concluíram que a estratégia cooperativa contribuiu para a construção de conhecimentos, além de aprimorar habilidades como fala, organização de ideias, escrita e resolução de conflitos. Já o trabalho de Marques, Ávila, Dias-Filho \& Silva (2016) utilizou diferentes estratégias cooperativas em uma intervenção de longa duração para o estudo das soluções químicas. Por meio de questionários e observação participante, os autores constataram que as estratégias cooperativas, de uma maneira geral, contribuíram para o aumento do desempenho individual.

O trabalho de Dos Santos, Crisostimo \& Komar (2017) foi voltado para alunos do $8^{\circ}$ ano do Ensino Fundamental e abordou a temática alimentação saudável. Os autores utilizaram o método de aprendizagem cooperativa STAD. Os alunos foram avaliados por meio de testes individuais, trabalhos escritos em grupo e apresentações das atividades realizadas em grupo. Os resultados revelaram que os desempenhos nos testes foram melhorando ao longo da intervenção e que apesar dos alunos terem enfrentado alguns problemas em relação as suas habilidades sociais, os grupos cooperativos tornaram a aula mais dinâmica e interessante. Há também relatos na literatura nacional do uso da aprendizagem cooperativa no ensino de Física. Leite, Lourenço, Licio \& Hernandes (2013) realizaram um minicurso de nanociência e nanotecnologia para 80 alunos do Ensino Médio, utilizando, dentre outros recursos, o método cooperativo Jigsaw. Os resultados mostraram que a utilização dessa estratégia de ensino cooperativo permitiu que os alunos ampliassem e correlacionassem os conceitos sobre o assunto, além de utilizarem da argumentação durante toda a dinâmica.

Apesar de todos os benefícios listados no presente trabalho, no que diz respeito a aquisição de conhecimentos e aprimoramento de habilidades, a incorporação da aprendizagem cooperativa no ensino de Ciências Naturais ainda apresenta desafios a serem superados. Zakaria \& Iksan (2006) destacam alguns problemas iniciais na utilização desse método nas aulas de Ciências e Matemática. Na visão dos autores, os professores precisam preparar materiais extras, uma vez que os materiais que os docentes têm disponível atualmente são, em sua maioria, de cunho individual. As atividades cooperativas levam mais tempo para serem desenvolvidas quando comparadas aos métodos tracionais. Assim, os professores podem se 
sentir receosos por não cobrir toda grade curricular. Eles ainda podem ter a descrença que os estudantes são capazes de aprender por conta própria, por isso entendem que a figura do professor ainda deve ser o centro do processo de ensino e aprendizagem. Os autores também ponderam que os professores não estão familiarizados com métodos de ensino cooperativo, já que provavelmente sua formação não os apresentou a tais metodologias de ensino (Zakaria \& Iksan, 2006). Não obstante aos desafios destacados, acredita-se que os ganhos proporcionados pela aprendizagem cooperativa em prol do ensino de Ciências Naturais superam e compensam os esforços para efetivar essa prática instrucional nas salas de aula.

\section{Considerações Finais}

As estratégias cooperativas colocam os alunos em uma posição ativa do conhecimento e emergem como uma alternativa as aulas tradicionais, nas quais o ensino é centralizado na figura do professor. Vale destacar que o ensino centralizado no aluno de forma alguma minimiza a figura do professor. $\mathrm{Na}$ aprendizagem cooperativa o professor exerce um papel fundamental. Ele determina quais são os objetivos do trabalho, a composição dos grupos, como as tarefas serão divididas, monitora o andamento do grupo e intervém para que os estudantes completem a tarefa e trabalhem em grupo de maneira eficiente. Além dos benefícios e da eficácia dessa prática instrucional, a aprendizagem cooperativa oferece aos estudantes a oportunidade de estudarem de forma criativa e dinâmica, proporcionando motivação para o aprendizado.

Apesar de todas as vantagens da utilização de estratégias cooperativas e da sua utilização em todo o mundo, poucos estudos no Brasil relatam o desenvolvimento de estratégias de ensino baseadas nessa metodologia. Sendo assim, as atividades cooperativas, adequadamente estruturadas, surgem como uma opção que pode e deve ser mais bem aproveitada pelos professores de disciplinas científicas na discussão de conteúdos de Ciências Naturais.

\section{Referências}

Acar, B., \& Tarhan, L. (2008). Effects of cooperative learning on students' understanding of metallic bonding. Research in Science Education, 38(4), 401-420.

Afonso, A. J. (2009). Nem tudo o que conta em educação é mensurável ou comparável. 
Crítica à accountability baseada em testes estandardizados e rankings escolares. Revista Lusofona de Educacao, (13), 13-29.

Anderson, W. L., Mitchell, S. M., \& Osgood, M. P. (2005). Comparison of student performance in cooperative learning and traditional lecture-based biochemistry classes. Biochemistry and Molecular Biology Education, 33(6), 387-393.

Armstrong, N., Chang, S.-M., \& Brickman, M. (2007). Cooperative learning in industrialsized biology classes. CBE - Life Sciences Education, 6, 163-171.

Aronson, E. (2002). Building empathy, compassion, and achievement in the jigsaw classroom. In J. Aronson (Ed.), Improving academic achievement: impact of psychological factors on education (pp. 209-225). San Diego, CA: Elsevier.

Colosi, J. C., \& Zales, C. R. (1998). Jigsaw cooperative learning improves biology lab courses. Bioscience, 48(2), 118-124.

Cross, D., Taasoobshirazi, G., Hendricks, S., \& Hickey, D. T. (2008). Argumentation: A strategy for improving achievement and revealing scientific identities. International Journal of Science Education, 30(6), 837-861.

Day, S. P., \& Bryce, T. G. K. (2013). The Benefits of Cooperative Learning to Socioscientific Discussion in Secondary School Science. International Journal of Science Education, 35(9), 1533-1560.

Dillenbourg, P. (1999). What do you mean by collaborative learning. Collaborative Learning: Cognitive and Computational Approaches, 1, 1-16. Retirado de: http://citeseerx.ist.psu.edu/viewdoc/download?doi=10.1.1.167.4896\&amp

Dos Santos, S. A., Crisostimo, A. L \& Komar, E. A. B. (2017). Aplicação do método de aprendizagem cooperativa STAD no ensino do tema alimentação saudável. Enseñanza de las ciencias, (Extra), 3991-3996.

Ebrahim, A. (2012). The effect of cooperative learning strategies on elementary students' 
(CC BY 4.0) | ISSN 2525-3409 | DOI: http://dx.doi.org/10.33448/rsd-v9i7.3611

science achievement and social skills in Kuwait. International Journal of Science and Mathematics Education, 10(2), 293-314.

Eymur, G., \& Geban, Ö. (2016). The Collaboration of Cooperative Learning and Conceptual Change : Enhancing the Students' Understanding of Chemical Bonding Concepts. International Journal of Science and Mathematics Education, 1-19.

Felder, Richard, M., \& Brente, R. (2007). Cooperative learning. In Active Learning Models from the Analytical Sciences (pp. 33-54).

Fatareli, E. F., Ferreira, L. N. D. A., Ferreira, J. Q., \& Queiroz, S. L. (2010). Método cooperativo de aprendizagem Jigsaw no ensino de cinética química. Química nova na escola, 32(3), 161-168.

Fernández-Santander, A. (2008). Cooperative learning combined with short periods of lecturing: A good alternative in teaching biochemistry. Biochemistry and Molecular Biology Education, 36(1), 34-38.

Genc, M. (2016). An evaluation of the cooperative learning process by sixth-grade students. Research in Education, 95(1), 19-32.

Gillies, R. M. (2016). Cooperative Learning: Review of Research and Practice. Australian Journal of Teacher Education, 41(3).

Gillies, R. M. (2014). Cooperative Learning: Developments in Research. International Journal of Educational Psychology, 3(2), 125-140.

Gillies, R. M., \& Nichols, K. (2015). How to Support Primary Teachers' Implementation of Inquiry: Teachers' Reflections on Teaching Cooperative Inquiry-Based Science. Research in Science Education, 45(2), 171-191.

Goodell, L. S., Cooke, N. K., \& Ash, S. L. (2012). Cooperative Learning Through In-Class Team Work: An Approach to Classroom Instruction in a Life Cycle Nutrition Course. NACTA Journal, 56(2), 68-75. 
Gupta, M., Jain, M., \& Pasrija, P. (2014). Gender related effects of co-operative learning strategies (Stad and Tai) on mathematics achievement. Issues and Ideas in Education, 2(March), 53-68.

Herreid, C. F. (1998). Why Isn’t Cooperative Learning Used to Teach Science? BioScience, 48(7), 553-559.

Ho, F. F., \& Boo, H. K. (2007). Cooperative learning: Exploring its effectiveness in the Physics classroom. Asia-Pacific Forum on Science Learning and Teaching, 8(2), 1-21. Hogan, K., Nastasi, B. K., \& Pressley, M. (1999). Discourse Patterns and Collaborative Scientific Reasoning in Peer and Teacher-Guided Discussions. Cognition and Instruction, 17(4), 379-432.

Hwong, N. C., Caswell, A., Johnson, D. W., \& Johnson, R. T. (1993). Effects of Cooperative and Individualistic Learning on Prospective Elementary Teachers' Music Achievement and Attitudes. The Journal of Social Psychology, 133(1), 53-64.

Johnson, D. W., \& Johnson, R. T. (1999). Making cooperative learning work. Theory into practice, $38(2), 67-73$.

Johnson, D. W., \& Johnson, R. T. (2009). An Educational Psychology Success Story: Social Interdependence Theory and Cooperative Learning. Educational Researcher, 38(5), 365-379.

Johnson, D. W., \& Johnson, R. T. (2013). International guide to student achievement $\left(1^{\mathrm{o}}\right.$ edição; J. Hattie \& E. M. Anderman, Eds.). Nova Iorque, UK: Taylor \& Francis.

Johnson, D. W., Johnson, R. T., \& Holubec, E. J. (1994). Cooperative learning in the classroom. Association for Supervision and Curriculum Development, 1250 N. Pitt St., Alexandria, VA 22314 (\$13.95, Stock Number: 1-94224).

Johnson, D. W., Johnson, R. T., \& Stanne, M. B. (2000). Cooperative Learning Methods: A Meta Analysis Cooperative Learning Methods: A Meta-Analysis. 
Johnson, D. W., Maruyama, G., Johnson, R., Nelson, D., \& Skon, L. (1981). Effects of cooperative, competitive, and individualistic goal structures on achievement: A meta-analysis. Psychological Bulletin, 89(1), 47-62.

King, A. (2002). Structuring Peer Interaction to Promote High-Level Cognitive Processing. Theory Into Practice, 41(1), 33-39.

Kirschner, F., Paas, F., Kirschner, P. A., \& Janssen, J. (2011). Differential effects of problemsolving demands on individual and collaborative learning outcomes. Learning and Instruction, 21(4), 587-599.

Laal, M. (2013). Positive Interdependence in Collaborative Learning. Procedia - Social and Behavioral Sciences, 93, 1433-1437.

LaCarrubba, A. (1993). A Comparative Study of the Academic Achievement of Primary Students When Learning Science through the Directed Reading Activity or Cooperative Learning Approach. Kean College of New Jersey.

Leite, I. S., Lourenço, A. B., Licio, J. G., \& Hernandes, A. C. (2013). Uso do método cooperativo de aprendizagem Jigsaw adaptado ao ensino de nanociência e nanotecnologia. Revista Brasileira de Ensino de Física, 35(4), 4504.

Li, M. P., \& Lam, B. H. (2013). Cooperative Learning. The Hong Kong Institute of Education, pp. 1-33.

Lord, T. R. (2001). 101 reasons for using cooperative learning in biology teaching. The American Biology Teacher, 63(1), 30-38.

Marques, S. P. D., Ávila, F. N., Dias Filho, F. A., \& Silva, M. G. V. (2016). Aprendizagem cooperativa como estratégia no aprendizado de química no ensino médio. Conexões-Ciência e Tecnologia, 9(4), 57-66.

Muraya, D. N., \& Kimamo, G. (2011). Effects of Cooperative Learning Approach on Biology Mean Achievement Scores of Secondary School Students' in Machakos District, Kenya. 
Educational Research and Reviews, 6(12), 726-745.

Oliveira, B. R. M., Kiouranis, N. M. M., Eichler, M. L., \& Queiroz, S. L. (2017).

Chocoquímica: construíndo conhecimento acerca do chocolate por meio do método de aprendizagem cooperativa Jigsaw. Química Nova na Escola, 39( 3), 277-285.

Panitz, T. (1999). Collaborative versus cooperative learning: a comparison of the two concepts which will help us understand the underlying nature of interactive learning.

Patchen, T., \& Smithenry, D. W. (2015). More Than Just Chemistry: The Impact of a Collaborative Participant Structure on Student Perceptions of Science. Research in Science Education, 45(1), 75-100. https://doi.org/10.1007/s11165-014-9414-1

Pereira, A.S. et al. (2018). Metodologia da pesquisa científica. [e-book]. Santa Maria. Ed. UAB/NTE/UFSM. Disponível em:

https://repositorio.ufsm.br/bitstream/handle/1/15824/Lic_Computacao_MetodologiaPesquisa-Cientifica.pdf?sequence=1. Acesso em: 30 Abril 2020.

Rothenberg, J. J., McDermott, P., \& Martin, G. (1998). Changes in Pedagogy: A Qualitative Result of Teaching Heterogeneous Classes. Teaching and Teacher Education, 14(6), 633642.

Silva, G. B. D., Teodoro, D. L., \& Queiroz, S. L. (2019). Aprendizagem cooperativa no ensino de ciências: uma revisão da literatura. Investigações em Ensino de Ciências, 24(3), 130.

Slavin, R. E. (1989). Cooperative learning and student achievement. In R. E. Slavin (Ed.), School and classroom organization (pp. 129-168). Nova Iorque e Londres: Taylor \& Francis.

Slavin, R. E. (2010). Co-operative learning: what makes group-work work? In F. B. Hanna Dumont, David Istance (Ed.), The Nature of Learning: using research to inspire practice (pp. 161-178). OECD.

Slavin, R. E. (2015). Cooperative Learning in Elementary Schools. Education 3-13, 43(1), 5- 
14. https://doi.org/10.1080/03004279.2015.963370

Tanner, K., Chatman, L. S., \& Allen, D. (2003). Approaches to cell biology teaching: cooperative learning in the science classroom--beyond students working in groups. Cell Biology Education, 2(1), 1-5.

Tanner, Kimberly, Chatman, L. S., \& Allen, D. (2003). Approaches to cell biology teaching: cooperative learning in the science classroom--beyond students working in groups. Cell Biology Education, 2(1), 1-5.

Theodoro, F. C. M., Costa, J. B. de S., \& Almeira, L. M. de. (2015). Modalidades e recursos didáticos mais utilizados no ensino de Ciências e Biologia. Estação Científica (UNIFAP), $5(1), 127-139$.

Thomas, M. V., \& Kothari, R. G. (2015). A study on the effectiveness of a strategy based on cooperative learning for science teaching in class VII. MIER Journal of Educational Studies, Trends \& Practices, 5(2), 123-136.

Torres, P. L., \& Irala, E. A. F. (2007). Aprendizagem Colaborativa (Vol. 1, pp. 65-97). Vol. 1, pp. 65-97. Curitiba: SENAR-PR.

Zakaria, E., \& Iksan, Z. (2006). Promoting cooperative learning in science and mathematics education: A Malaysian perspective. Eurasia Journal of Mathematics, Science \& Technology Education, 3(1), 35-39.

\section{Porcentagem de contribuição de cada autor no manuscrito}

Cássio Gomes Rosse - 60\%

Leandra Marques Chaves Melim - 40\% 